\title{
Fiber to the Premise (FTTP) Industry Structure: Implications of a Wholesale-Retail Split
}

\author{
Anupam Banerjee \\ Department of Engineering \& Public Policy \\ Carnegie Mellon University \\ Pittsburgh, PA 15213 USA \\ anupam_banerjee@cmu.edu
}

\author{
Marvin Sirbu \\ Department of Engineering \& Public Policy \\ Carnegie Mellon University \\ Pittsburgh, PA 15213 USA \\ sirbu@cmu.edu
}

\begin{abstract}
In some communities, we observe a single vertically integrated FTTP network owner and service provider that can either be profit maximizing or welfare maximizing. In other cases, either out of choice or regulation, we observe the network owner (the wholesaler) leasing facilities to competing service providers (retailers), who then provide voice, video and data service over the shared network (wholesale-retail split). The network owner can either wholesale dark fiber or "lit" transport (and can be profit maximizing or welfare maximizing). In this paper, using a simplified model where subscribers always buy voice service and choose between either broadband data service or video service or the video and data bundle, we show that such a wholesale-retail split interferes with the ability of the network owner to price discriminate. We conclude that for a bivariate correlated normal distribution of willingness to pay for data and video services, the supply side economics of FTTP (i.e., the fact that the marginal cost of provisioning the bundle is less than the sum of the marginal costs of provisioning the individual services) ensures that the bulk of the extractable economic surplus resides in the bundle and not in the individual services. Since the wholesale-retail split interferes with wholesaler's ability to extract economic surplus only from an individual service (video service) and not from the bundle, the implications (the difference between the profits of a profit maximizing wholesaler and a profit maximizing vertically integrated entity) are modest, at best. Our analysis also shows that municipalities or communities that build out FTTP and choose to be wholesalers (i) can realize sustainable prices, (ii) are likely to create greater welfare and (iii) are just as likely to recover costs (vis-à-vis vertically integrated entities).
\end{abstract}

\section{INTRODUCTION}

Our earlier research has shown that the Fiber to the Premise (FTTP) industry has natural monopoly characteristics [1], implying that it may not be economically feasible to have more than one physical FTTP network to serve a particular market or community. However, service level (or non-facilities based) competition is possible in the FTTP industry [1] - competition that can be achieved by way of a 'wholesale-retail split', that is, a structural separation (by either regulation or choice) between infrastructure ownership and (voice, video and data) service provisioning. In this work, we examine the implications of such a wholesale retail split for producer profits and consumer surplus - more specifically we seek to understand if it is feasible (from an economic standpoint only) to impose structural separation between infrastructure ownership and service provisioning in FTTP. In section II, we further formalize the notion of the wholesale-retail split by laying out five potential FTTP industry structures. The demand and supply models used for welfare computations are introduced in sections III and IV respectively. The theoretical model of the wholesale-retail split is presented in section V. We conclude with some empirical results for the simplified model (section VI) and caveats and future work (section VII).

\section{FTTP INDUSTRY STRUCTURES}

In some communities, we observe a single vertically integrated FTTP network owner and (voice, video and data) retail service provider, that can either be profit maximizing (henceforth referred to as the 'Verizon' model) or welfare maximizing (henceforth referred to as the 'Bristol, VA' model [8]). In contrast, either out of choice or due to regulation, we observe the network owner (henceforth referred to as the 'wholesaler') leasing facilities to competing service providers (retailers), who then provide voice, video and data service over the shared network (wholesale-retail split). For example, consider a network owned by Grant County, WA (figure 1) with Qwest providing voice services, Comcast providing video services and AOL providing broadband data services over it. The network owner can either sell dark fiber (henceforth referred to as the 'Stockholm' model) or 'lit' transport (henceforth referred to as the 'Grant-County-Profit' model or 'Grant-County-Welfare' model, depending on whether the wholesaler is profit or welfare maximizing).

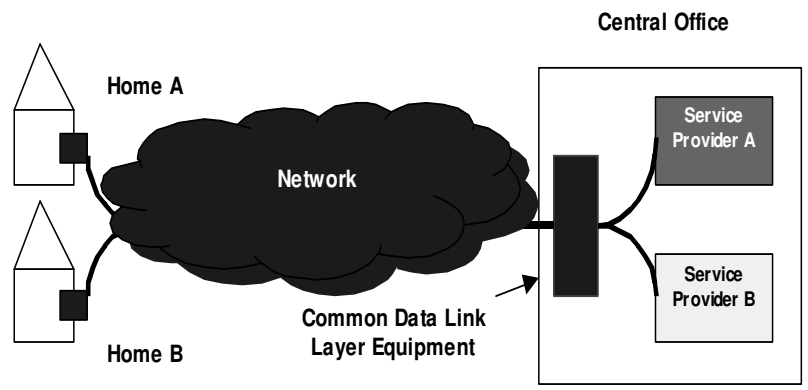

Figure 1. Wholesale Retail Split

This research was sponsored by the NSF under ITR Award ANI-0331653. 


\section{DEMAND MODEL}

In order to compute producer profits and consumer surplus and compare them across the five different industry structures we need to construct models for demand and supply. Consider a 3-space, where the coordinates of each point give the willingness to pay for voice, data and video services for a particular home. For a preliminary analysis, we assume that every home takes voice services; thereby reducing the demand model to 2-space, where the coordinates of each point give the willingness to pay for data service and video service respectively. Let $x_{1}$ be the willingness to pay for data service and $x_{2}$ the willingness to pay for video service for a particular home: notice that $x_{1}$ and $x_{2}$ may be correlated. We shall assume the values of $x_{1}$ and $x_{2}$ for each household are drawn from a correlated bivariate normal distribution-i.e. the probability that a particular home has willingness to pay $x_{1}$ for data and $x_{2}$ for video is given by the bivariate normal probability density function [6]:

$$
P\left(x_{1}, x_{2}\right)=\frac{1}{2 \pi \sigma_{1} \sigma_{2} \sqrt{1-\rho^{2}}} \exp \left[-\frac{z}{2\left(1-\rho^{2}\right)}\right]
$$

where,

$$
z=\frac{\left(x_{1}-\mu_{1}\right)^{2}}{\sigma_{1}{ }^{2}}-\frac{2 \rho\left(x_{1}-\mu_{1}\right)\left(x_{2}-\mu_{2}\right)}{\sigma_{1} \sigma_{2}}+\frac{\left(x_{2}-\mu_{2}\right)^{2}}{\sigma_{2}{ }^{2}}
$$

and,

$$
\mu_{1}=\text { mean willingness to pay for data service }
$$

$\mu_{2}=$ mean willingness to pay for video service

$\sigma_{1}=$ standard deviation of willingness to pay for data service

$\sigma_{2}=$ standard deviation of willingness to pay for data service

$\rho=$ coefficient of correlation between the willingness to pay for data and video service respectively for a particular home.

A homeowner can choose to purchase (i) no service, (ii) data service only, (iii) video service only, or (iv) a bundle of data and video service. We define net utility of a transaction as the difference between willingness to pay and price. A homeowner will choose to make a purchase only if it has a both a positive net utility and a greater net utility than the other two possible transactions.[3]. More formally, if a service provider sets price $P_{1}$ for data service, $P_{2}$ for video service and $P_{12}$ for the bundle, a home prefers data service only if,

$$
\begin{aligned}
& x_{1}-P_{1} \geq 0 \ldots \ldots \ldots \ldots \\
& x_{1}-P_{1} \geq x_{2}-P_{2} \ldots \ldots \ldots \\
& x_{1}-P_{1} \geq x_{1}+x_{2}-P_{12}
\end{aligned}
$$

From (1), (2) and (3),

$$
\begin{aligned}
& x_{1} \geq P_{1} \ldots \ldots \ldots \\
& x_{2} \leq P_{12}-P_{1} .
\end{aligned}
$$

Therefore, if $Q_{1}$ is the proportion of subscribers that take data service,

$$
Q_{1}=\int_{-\infty}^{P_{12}-P_{1}} \int_{P_{1}}^{\infty} P\left(x_{1}, x_{2}\right) d x_{1} d x_{2}
$$

Similarly, if $Q_{2}$ is the proportion of subscribers that take video service,

$$
Q_{2}=\int_{-\infty}^{P_{12}-P_{2}} \int_{P_{2}}^{\infty} P\left(x_{1}, x_{2}\right) d x_{2} d x_{1} \ldots \ldots \ldots(7)
$$

Using similar arguments, a home prefers the bundle over the individual services only if,

$$
\begin{aligned}
& x_{1}+x_{2}-P_{12} \geq 0 \\
& x_{1}+x_{2}-P_{12} \geq x_{1}-P_{1} . \\
& x_{1}+x_{2}-P_{12} \geq x_{2}-P_{2}
\end{aligned}
$$

From (8), (9) and (10),

$$
\begin{aligned}
& x_{1}+x_{2} \geq P_{12} . \\
& x_{2} \geq P_{12}-P_{1} . \\
& x_{1} \geq P_{12}-P_{2} .
\end{aligned}
$$

Therefore, if $Q_{12}$ is the proportion of subscribers that take data service,

$$
\begin{aligned}
& Q_{12}=\int_{P_{12}-P_{1}}^{\infty} \int_{P_{12}-P_{2}}^{\infty} P\left(x_{1}, x_{2}\right) d x_{1} d x_{2} \\
& -\int_{P_{12}-P_{1}}^{P_{2}} \int_{P_{12}-x_{2}}^{P_{1}} P\left(x_{1}, x_{2}\right) d x_{1} d x_{2} \ldots \ldots . .
\end{aligned}
$$

Figure 2 shows a distribution of consumers who have a mean willingness to pay of $\$ 35$ per month for data $\left(\mu_{1}\right), \$ 45$ per month for video $\left(\mu_{2}\right)$ and a coefficient of correlation $(\rho)$ of $(-$ $0.5)$; the areas $B D P_{1} P_{3}, A C P_{2} P_{3}, A C D B Z$ correspond to the fraction (or proportion) of homes that take data service $\left(Q_{1}\right)$, video service $\left(Q_{2}\right)$ and the bundle $\left(Q_{12}\right)$ respectively.

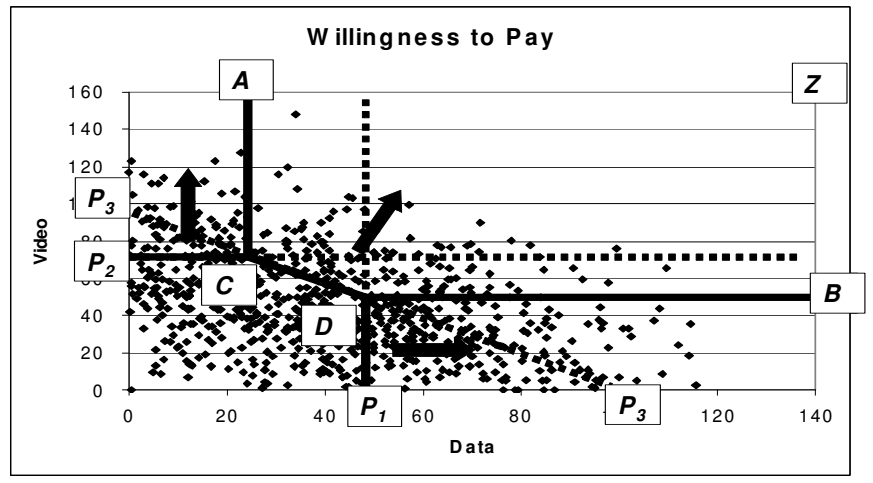

Figure 2. Distribution of Willingness to Pay for Data and Video services 


\section{SUPPLY MODEL}

Regardless of how many homes subscribe for any of the services, the network owner has to build out the network to all the $Q$ homes in a market, incurring an annualized (sunk) cost of $F$ [7]. Once a home decides to take any service, the network owner (or retail service provider in the 'Stockholm' model) has to deploy the drop loop and provision central office and customer premises equipment at an annualized cost of $C_{0}$.

It is further assumed that the annual incremental cost of providing data service is $C_{1}$ per home served. This includes the cost of transit, second mile costs of transporting data from the central office to the point of presence of the Internet backbone provider and operations and marketing costs. Providing video services requires setting up a video head end and purchasing rights to content. These costs, in addition to costs of second mile transport from the central office to the head end and costs of operations and marketing constitute the annual incremental cost of providing video service, which is assumed to be $C_{2}$ per home served. Clearly the marginal cost of serving a data customer is $C_{0}+C_{1}$, that of serving a video customer is $C_{0}+C_{2}$ and that of provisioning a bundle is $C_{0}+C_{1}+C_{2}$. Since, $\left(C_{0}+\right.$ $\left.C_{1}+C_{2}\right)<\left(C_{0}+C_{1}\right)+\left(C_{0}+C_{2}\right)$, the marginal cost of supplying the bundle is always less than the sum of the marginal costs of providing the individual services. (It is possible that the incremental costs of providing a bundle are less than the sum of the incremental costs for each of the individual services-due, for example, to economies of scope in operations or marketing-but we ignore this possibility for the present analysis; such economies would only reinforce our conclusions.)

The total cost (per home passed) of providing data service to (a proportion of) $Q_{1}$ homes, video service to (a proportion of) $Q_{2}$ homes and the bundle to (a proportion of) $Q_{12}$ homes is:

$C=\frac{F}{Q}+\left(C_{0}+C_{1}\right) Q_{1}+\left(C_{0}+C_{2}\right) Q_{2}+\left(C_{0}+C_{1}+C_{2}\right) Q_{12}$

\section{THEORETICAL MODELS FOR INDUSTRY STRUCTURES}

\section{A. Vertically Integrated Industry Structures}

'Verizon', being a vertically integrated monopolist, can sell three goods to consumers: data service, video service or the bundle and can therefore set three prices to maximize its profit function. More formally, if 'Verizon' sets a price $P_{1}$ for data, $P_{2}$ for video and $P_{12}$ for the bundle, and serves (a proportion of) $Q_{1}$ data customers (or homes), (a proportion of) $Q_{2}$ video customers (or homes) and (a proportion of) $Q_{12}$ bundle customers (or homes), its revenue per home passed $(R)$, cost per home passed $(C)$ and profit (per home passed) functions are:

$$
\begin{aligned}
& R=P_{1} Q_{1}+P_{2} Q_{2}+P_{12} Q_{12} \\
& C=\frac{F}{Q}+\left(C_{0}+C_{1}\right) Q_{1}+\left(C_{0}+C_{2}\right) Q_{2}+\left(C_{0}+C_{1}+C_{2}\right) Q_{12} \\
& \text { Profit }=\frac{F}{Q}+\left(P_{1}-C_{0}-C_{1}\right) Q_{1}+\left(P_{2}-C_{0}-C_{2}\right) Q_{2}+\left(P_{12}-C_{0}-C_{1}-C_{2}\right) Q_{12}
\end{aligned}
$$

where, $Q_{1}, Q_{2}$ and $Q_{12}$ are given by equations (6), (7) and (14). Since, all prices are positive and the price of the bundle can never exceed the sum of the prices of the individual services, the optimization problem faced by 'Verizon' can be written as

$$
\begin{aligned}
& \underset{P_{1}, P_{2}, P_{12}}{\operatorname{Maximize}} \operatorname{Pr} \text { ofit } \\
& \text { s.t., } \\
& P_{1}, P_{2}, P_{12} \geq 0 \\
& P_{1}+P_{2} \geq P_{12}
\end{aligned}
$$

'Bristol', which is a welfare maximizing vertically integrated monopolist, has revenue and cost functions that are identical to 'Verizon', but instead chooses $P_{1}, P_{2}$ and $P_{12}$ to maximize consumer surplus. The consumer surplus associated with data service per home passed $\left(C S_{1}\right)$, video service per home passed $\left(C S_{2}\right)$ and the bundle per home passed $\left(C S_{12}\right)$ are given by:

Since, all prices are positive and the price of the bundle can never exceed the sum of the prices of the individual services, the optimization problem faced by 'Bristol' can be written as:

$$
\begin{aligned}
& \underset{P_{1}, P_{2}, P_{12}}{\text { Maximize ConsumerSurplus }} \\
& \text { s.t., } \\
& P_{1}, P_{2}, P_{12} \geq 0 \\
& P_{1}+P_{2} \geq P_{12}
\end{aligned}
$$

\section{B. Implications of a Wholesale-Retail Split}

By virtue of the fact that they are vertically integrated, both 'Verizon' and 'Bristol' can engage in third degree price discrimination [2]. While 'Grant County' (a wholesaler that sells layer 2 'lit' transport services to retail service providers) can potentially sell a data capability service1, a video capability service $^{2}$ and a bundle capability service, due to the bursty nature IP data traffic and by the virtue of statistical multiplexing, a video capability service has sufficient bandwidth to support the bundle. Therefore, once a wholesaler sells a video capability to a retail service provider, in effect it also sells a bundle capability because a retailer can provision a bundle over the video capability without the wholesaler's explicit knowledge. A wholesaler can only sell two goods: (i) a data capability and (ii) a video (or bundle) capability; hence, it can set only two prices. Clearly, a wholesale-retail split interferes with the ability of a network owner to price discriminate.

\footnotetext{
${ }^{1}$ By data capability we mean a certain amount of bandwidth that the wholesaler sells and is sufficient to support a retail broadband data service offering. For example, let us say that a data capability is a full duplex $1 \mathrm{Mbps}$ layer 2 capacity.

${ }^{2}$ Let us say that a video capability service is a full duplex 4 Mbps layer 2 capacity. So that we can shed light on the implications of a wholesale retail split with a simplified 2-service model, we assume that the wholesaler does not sell asymmetric capacity.
} 


$$
\begin{aligned}
& C S_{1}=\int_{-\infty}^{P_{12}-P_{1}} \int_{P_{1}}^{\infty}\left(x_{1}-P_{1}\right) P\left(x_{1}, x_{2}\right) d x_{1} d x_{2} \\
& C S_{2}=\int_{-\infty}^{P_{12}} \int_{P_{2}}^{\infty}\left(x_{2}-P_{2}\right) P\left(x_{1}, x_{2}\right) d x_{2} d x_{1} \\
& C S_{12}=\int_{P_{12}-P_{1}}^{\infty} \int_{12}^{\infty}\left(x_{1}+x_{2}-P_{12}\right) P\left(x_{1}, x_{2}\right) d x_{1} d x_{2} \\
& -\int_{P_{12}-P_{1}}^{P_{2}} \int_{P_{12}-x_{2}}^{P_{1}}\left(x_{1}+x_{2}-P_{12}\right) P\left(x_{1}, x_{2}\right) d x_{1} d x_{2} \\
& \text { ConsumerSu rplus }=C S_{1}+C S_{2}+C S_{12}
\end{aligned}
$$

\section{Wholesale-Retail Split Industry Structures}

'Grant County' can set two prices $P_{01}$ and $P_{02}$ for data capability and video (or bundle) capability respectively. We assume that the retail industry is perfectly competitive and therefore retail service providers make no profit. A retailer can buy a data capability from Grant County for $P_{01}$ and sell a retail data offering for $P_{1}=P_{01}+C_{1}$. Similarly, a retailer can buy a video (or bundle) capability from Grant County for $P_{02}$ and sell a retail video offering for $P_{2}=P_{02}+C_{2}$ or a retail bundle offering for $P_{12}=P_{02}+C_{1}+C_{2}$. Notice that due to the wholesale-retail split, the retail price of the bundle exceeds the retail price of the video service by exactly the incremental cost of providing data service (that is, $P_{12}=P_{2}+C_{1}$ ).

If $Q_{1}$ (is the proportion of) homes (that) take data service, $Q_{2}$ (is the proportion of) homes (that) take video service and $Q_{12}$ (is the proportion of) homes (that) take the bundle, 'Grant County Profit' generates a revenue (per home passed) of $R=$ $P_{01} Q_{1}+P_{02}\left(Q_{2}+Q_{12}\right)$ and incurs a total cost (per home passed) of $C=F / Q+C_{0}\left(Q_{1}+Q_{2}+Q_{12}\right)$. Since 'Grant County' is a wholesaler providing layer 2 transport services, it does not incur any costs related to providing data or video services. The profit (per home passed) function of 'Grant County Profit' is given by

$$
\text { Profit }=\left(P_{01}-C_{0}\right) Q_{1}+\left(P_{02}-C_{0}\right)\left(Q_{2}+Q_{12}\right)-F / Q
$$

Where, $Q_{1}, Q_{2}$ and $Q_{12}$ are given by

$$
\begin{aligned}
& Q_{1}=\int_{-\infty}^{P_{02}-P_{01}+C_{2}} \int_{P_{01}+C_{0}+C_{1}}^{\infty} P\left(x_{1}, x_{2}\right) d x_{1} d x_{2} \\
& Q_{2}=\int_{-\infty}^{C_{1}} \int_{P_{02}}^{\infty} P\left(x_{1}, x_{2}\right) d x_{2}+C_{2} d x_{1} \\
& Q_{12}=\int_{P_{02}}^{\infty} \int_{P_{01}+C_{2}} P\left(x_{1}, x_{2}\right) d x_{1} d x_{2} \\
& -\int_{P_{01}}^{\infty}+C_{0}+C_{1} \\
& \left.P_{02}-P_{01}+C_{2} P_{02}+C_{0}+C_{1}+C_{2}-x_{2}, x_{2}\right) d x_{1} d x_{2}
\end{aligned}
$$

Since, all prices are positive and the price of the data capability can never exceed the price of the video (or bundle) capability $^{3}$, the optimization problem faced by 'Grant County Profit' can be written as

$$
\begin{aligned}
& \underset{P_{01}, P_{02}}{\operatorname{Maximize}} \operatorname{Pr} \text { ofit } \\
& \text { s.t., } \\
& P_{01}, P_{02} \geq 0 \\
& P_{02} \geq P_{01}
\end{aligned}
$$

'Grant County Welfare' has identical revenue and cost functions as 'Grant County Profit', but instead chooses $P_{01}$ and $P_{02}$ to maximize consumer surplus. The consumer surplus (per home passed) associated with data service $\left(C S_{1}\right)$, video service $\left(C S_{2}\right)$ and the bundle $\left(C S_{12}\right)$ are given by:

$$
\begin{aligned}
& C S_{1}=\int_{-\infty}^{P_{02}-P_{01}+C_{2}} \int_{P_{01}+C_{0}+C_{1}}^{\infty}\left(x_{1}-P_{01}-C_{0}-C_{1}\right) P\left(x_{1}, x_{2}\right) d x_{1} d x_{2} \\
& C S_{2}=\int_{-\infty P_{02}+C_{0}+C_{2}}^{C_{1}} \int_{02}^{\infty}\left(x_{2}-P_{02}-C_{0}-C_{2}\right) P\left(x_{1}, x_{2}\right) d x_{2} d x_{1} \\
& C S_{12}=\int_{P_{02}-P_{01}+C_{2}}^{\infty} \int_{C_{1}}^{\infty}\left(x_{1}+x_{2}-P_{02}-C_{0}-C_{1}-C_{2}\right) P\left(x_{1}, x_{2}\right) d x_{1} d x_{2} \\
& -\int_{P_{02}-P_{01}+C_{2}}^{P_{02}+C_{0}+C_{1}+C_{2}-x_{2}} \int_{P_{01}+C_{0}+C_{1}}^{P_{2}}\left(x_{1}+x_{2}-P_{02}-C_{0}-C_{1}-C_{2}\right) P\left(x_{1}, x_{2}\right) d x_{1} d x_{2} \\
& \text { ConsumerSurplus }=C S_{1}+C S_{2}+C S_{12}
\end{aligned}
$$

Since, all prices are positive and the price of the data capability can never exceed the price of the video (or bundle) capability, the optimization problem faced by 'Grant County Welfare' can be written as

$$
\begin{aligned}
& \underset{P_{01}, P_{02}}{\text { Maximize ConsumerSurplus }} \\
& \text { s.t., } \\
& P_{01}, P_{02} \geq 0 \\
& P_{02} \geq P_{01}
\end{aligned}
$$

'Stockholm' sells only one good: dark fiber access from the Central office to each home; hence it has the least 'pricing flexibility' among all the industry structures and can set only one price $P_{0}$. We again assume that the retail industry is perfectly competitive and therefore all retail service providers make no profit. A retailer can buy the dark fiber from 'Stockholm' for $P_{0}$ and sell a retail data offering for $P_{1}=P_{0}+$ $C_{0}+C_{1}$, a retail video offering for $P_{2}=P_{0}+C_{0}+C_{2}$ or a retail bundle offering for $P_{12}=P_{0}+C_{0}+C_{1}+C_{2}$. Notice that due to 'Stockholm's decision to sell only dark fiber, the retail price of the bundle exceeds the retail price of the video service by exactly the incremental cost of providing data service (that is, $P_{12}=P_{2}+C_{1}$ ), while the retail price of the bundle exceeds the retail price of the data service by exactly the incremental cost of providing video service (that is, $P_{12}=P_{1}+C_{2}$ ).

'Stockholm' generates a revenue (per home passed) of $R=$ $P_{0}\left(Q_{1}+Q_{2}+Q_{12}\right)$ and incurs a total cost (per home passed) of $C=F / Q$. Since 'Stockholm' is a 'dark fiber' wholesaler, it does

\footnotetext{
${ }^{3}$ Otherwise retail service providers would provision data service over the cheaper video capability
} 
not incur any costs related to the provisioning of the customer premises equipment, the central office equipment, the drop loop and providing data or video services. The profit (per home passed) function of 'Stockholm' is given by

Profit $=P_{0}\left(Q_{1}+Q_{2}+Q_{12}\right)-F / Q$

Where, $Q_{1}, Q_{2}$ and $Q_{12}$ are given by

$$
\begin{aligned}
& Q_{1}=\int_{-\infty P_{0}+C_{0}+C_{1}}^{C_{2}} \int_{2}^{\infty} P\left(x_{1}, x_{2}\right) d x_{1} d x_{2} \\
& Q_{2}=\int_{-\infty P_{0}+C_{0}+C_{2}}^{C_{1}} P\left(x_{1}, x_{2}\right) d x_{2} d x_{1} \\
& Q_{12}=\int_{C_{2}}^{\infty} \int_{C_{1}}^{\infty} P\left(x_{1}, x_{2}\right) d x_{1} d x_{2}-\int_{C_{2}}^{P_{0}+C_{0}+C_{2}} \int_{P_{0}+C_{0}+C_{1}+C_{2}-x_{2}}^{P_{0}+C_{0}+C_{1}} P\left(x_{1}, x_{2}\right) d x_{1} d x_{2}
\end{aligned}
$$

The consumer surplus (per home passed) associated with data service $\left(C S_{1}\right)$, video service $\left(C S_{2}\right)$ and the bundle $\left(C S_{12}\right)$ are given by:

$$
\begin{aligned}
& C S_{1}=\int_{-\infty P_{0}+C_{0}+C_{1}}^{C_{2}} \int_{2}^{\infty}\left(x_{1}-P_{0}-C_{0}-C_{1}\right) P\left(x_{1}, x_{2}\right) d x_{1} d x_{2} \\
& C S_{2}=\int_{-\infty P_{0}+C_{0}+C_{2}}^{C_{1}} \int_{2}^{\infty}\left(x_{2}-P_{0}-C_{0}-C_{2}\right) P\left(x_{1}, x_{2}\right) d x_{2} d x_{1} \\
& C S_{12}=\int_{C_{2} C_{1}}^{\infty} \int_{1}^{\infty}\left(x_{1}+x_{2}-P_{0}-C_{0}-C_{1}-C_{2}\right) P\left(x_{1}, x_{2}\right) d x_{1} d x_{2} \\
& -\int_{C_{2}}^{P_{0}+C_{0}+C_{2}} \int_{P_{0}+C_{0}+C_{1}}^{P_{0}+C_{0}+C_{1}+C_{2}-x_{2}}
\end{aligned}
$$$$
\text { ConsumerSuplus }=C S_{1}+C S_{2}+C S_{12}
$$

We assume that 'Stockholm' is not a profit maximizing monopolist, because it can clearly generate more profit by following the 'Grant County Profit' model. Obviously, it can create more welfare by following the 'Grant County Welfare' model too, but it is conceivable that as a city trying to facilitate economic development, it just wants to build out the fiber infrastructure (like the water infrastructure, say) and not get into the business of 'lighting' it. Since, prices are positive, the optimization problem faced by 'Stockholm' can be written as

$$
\begin{aligned}
& \underset{P_{0}}{\text { Maximize ConsumerSurplus }} \\
& \text { s.t., } \\
& P_{0} \geq 0
\end{aligned}
$$

If $P_{1}{ }^{\mathrm{v}}, P_{2}{ }^{\mathrm{v}}$ and $P_{12}{ }^{\mathrm{v}}$ are the respective retail prices for data, video and the bundle in the vertically integrated industry structures (say Verizon), and $P_{1}, P_{2}$ and $P_{12}$ are the respective retail prices of data, video and the bundle in 'Grant County Profit' (say), figure 3 graphically shows the implications of the wholesale retail split. The pricing inflexibility imposed by the wholesale retail split ensures that the price of the retail video service is much higher in 'Grant County Profit' vis-à-vis 'Verizon' ensuring that 'Grant County Profit' has a lower number of video-only customers.

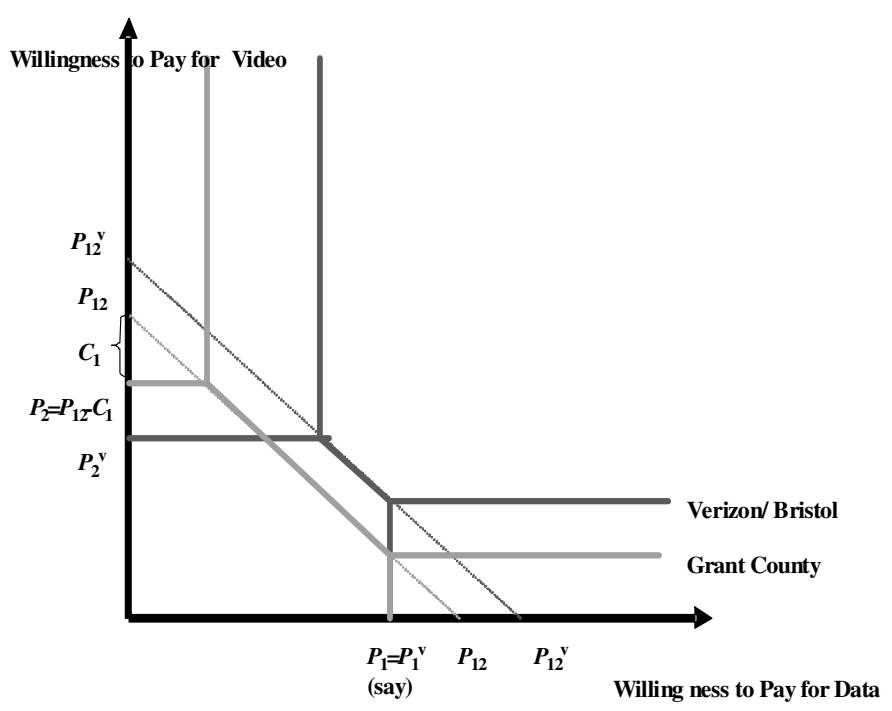

Figure 3. Implications of a Wholesale Retail Split

Similarly, the pricing inflexibility arising from 'Stockholm's decision of selling only dark fiber ensures that the price of the retail data and retail video services is much higher in 'Stockholm' vis-à-vis 'Verizon' ensuring that 'Stockholm' has a lower number of data-only and video-only customers. We observe the welfare implications of this pricing inflexibility in the next section.

\section{EMPIRICAL RESULTS}

For the base case, we assume that the network serves an urban market that has 10,000 homes and is characterized by the following model parameters.

\begin{tabular}{|l|l|l|}
\hline Parameter & Value & Explanation \\
\hline$Q$ & 10,000 homes & \\
\hline$\mu_{1}$ & $\$ 35 /$ home/ month & \\
\hline$\mu_{2}$ & $\$ 45 /$ home/ month & \\
\hline$\sigma_{1}$ & $\$ 10 /$ home/ month & \\
\hline$\sigma_{2}$ & $\$ 10 /$ home/ month & \\
\hline$F$ & $\$ 50,000 /$ month & $\begin{array}{l}\text { We have assumed a capital cost of } \$ 800 \\
\text { per home for installation of the fiber } \\
\text { resulting in a total capital expenditure of } \\
\$ 8 \text { million (amortized over 25 years at 5\% } \\
\text { cost of capital) }\end{array}$ \\
\hline$C_{0}$ & $\$ 8 /$ home/ month & $\begin{array}{l}\text { We have assumed a capital cost of } \$ 200 \\
\text { per home for installation of the drop } \\
\text { (amortized over 25 years at 5\% cost of } \\
\text { capital) and a cost of } \$ 400 \text { for OLT and } \\
\text { CPE (amortized it over 5 years at 5\% cost } \\
\text { of capital) }\end{array}$ \\
\hline$C_{1}$ & $\$ 20 /$ home/ month & \\
\hline$C_{2}$ & $\$ 30 /$ home/ month & \\
\hline$\rho$ & $-1<\rho<+1$ & \\
\hline
\end{tabular}

Figure 4. Parameter values for the base model

Figure 5 shows that the total welfare per home passed (sum of consumer and producer surplus) generated by the welfare maximizing industry structures ('Bristol', 'Grant County Welfare' and 'Stockholm') is higher than the total welfare (per home passed) generated by the profit maximizing counterparts 
'Verizon' and 'Grant County Profit' by about $\$ 2-\$ 5$ per month per home. It is interesting to note that for values of $\rho$ higher than 0.75 , all the welfare maximizing industry structures create almost the same amount of total welfare (and similarly the profit maximizing industry structures create almost an identical amount of total welfare). This is due to the fact that for values of $\rho$ above 0.75 , most customers seek the bundle. Since the wholesale retail split does not interfere with the ability of the wholesaler to extract the economic surplus ${ }^{4}$ associated with the bundle, the outcomes are very similar for 'Verizon' and 'Grant County Profit' and for 'Grant County Welfare', 'Bristol' and 'Stockholm'.

For other values of $\rho$, 'Bristol' generates greater welfare (per home passed) than 'Grant County Welfare' by about $\$ 0.60$ per month per home, while, 'Grant County Welfare' creates only marginally higher welfare per home passed (less than $\$ 0.10$ per month per home) vis-à-vis 'Stockholm'. The welfare difference between 'Bristol' and 'Grant County Welfare' increases as $\rho$ decreases because 'Bristol' serves many more video subscribers (than 'Grant County Welfare') due to the ability to set the video price independently of the bundle price. The welfare experienced by an increasing number of video subscribers at a lower video price widens the gap as $\rho$ decreases from 0.75 to -1 . Finally, figure 5 also shows that 'Verizon' creates marginally higher welfare (less than $\$ 0.10$ per month per home) over 'Grant County Profit'.

These observations have two very important policy implications: (i) Municipalities that are considering building out FTTP to maximize welfare can largely do so without being vertically integrated. Since 'Bristol' creates less than $\$ 1$ of additional welfare per home passed per month over 'Grant County Welfare' and 'Stockholm', it is conceivable that the additional welfare that subscribers experience due to increased product diversity and improved service resulting from retail competition (that our model does not capture) more than compensates for this modest welfare loss. (ii) FTTP network owners can realize sustainable wholesale prices and are almost as likely to recover costs as vertically integrated providers. This finding questions the 'popular' claim that the network owner has to be vertically integrated in order to be profitable and questions the assertion that a wholesale retail split does not provide the right incentives for investment. Since the difference between the total welfare outcomes of 'Verizon' and 'Grant County Profit' is about $\$ 0.10$ or less per home per month, profit maximizing municipalities that choose to be wholesalers are likely to create more total welfare, assuming that the additional welfare that subscribers experience by the virtue of retail competition more than compensates for $\$ 0.10$ welfare loss (per home passed).

\footnotetext{
${ }^{4}$ By surplus we refer to the area between the supply and the demand curves. Alternatively, this is also the maximum profit that can be extracted by a firm that has the ability to engage in first degree price discrimination.
}

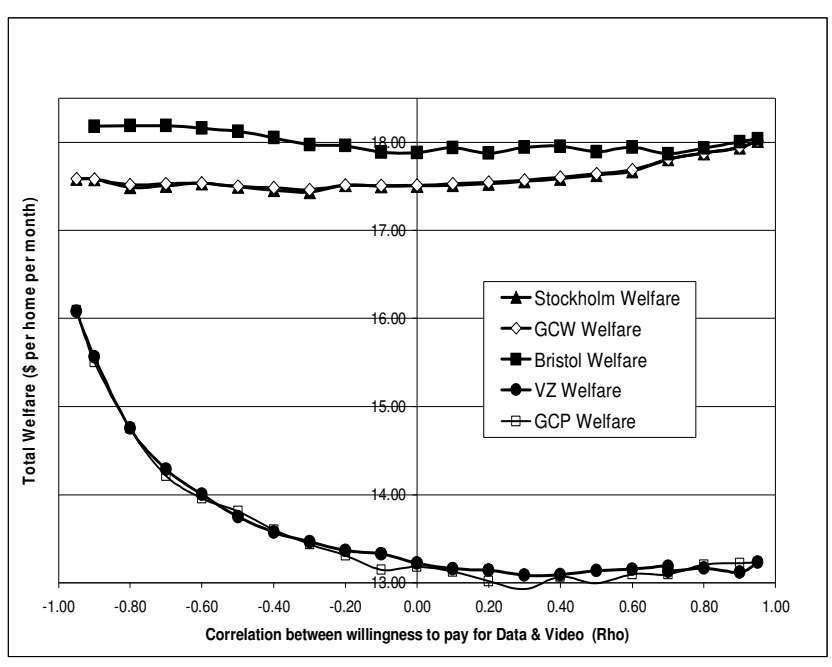

Figure 5. Total Welfare (per home passed) for all Industry Structures

'Verizon' and 'Grant County Profit' have very similar consumer surplus outcomes and marginally different profitability outcomes (Figure 6), in spite of having very different prices (Figure 7). The profit maximizing pricing strategy of 'Verizon' entails lowering the prices of the individual data and video services while increasing the price of the bundle as $\rho$ increases from -1 to +1 . Intuitively, this is because at values of $\rho$ closer to -1 , there are more users that seek the individual services (and hence higher prices for individual services are profit maximizing), while at values of $\rho$ closer to +1 more users seek the bundle (and hence a higher price for the bundle is profit maximizing).

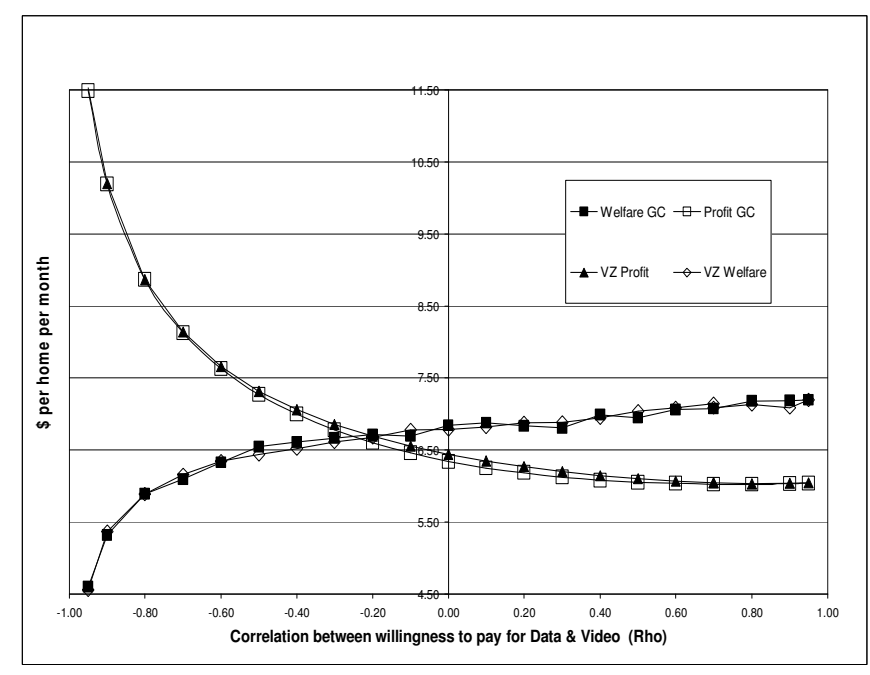

Figure 6. Comparison between 'Verizon' and 'Grant County Profit' (Profit per home passed and Consumer Surplus per home passed)

Figure 7 further shows that while the price of data set by both entities tends to be remarkably similar, 'Grant County Profit' has a lower bundle price (lower by about $\$ 0.20$ per home per month) but a much higher video price (higher by up to $\$ 8$ per home per month since it is tied to the bundle price). The consumer surplus outcomes are very similar because the decrease in consumer surplus from the video service (resulting 
from the much higher video price and fewer video subscribers) in the case of 'Grant County Profit' is roughly compensated by the increase in consumer surplus from the bundle (resulting from the marginally lower bundle price). Consumer Surplus always increases with $\rho$ because as $\rho$ goes to +1 , there are a lot more homes with high willingness to pay. Since consumer surplus experienced by a particular home is the difference between its willingness to pay and the price, homes with high willingness to pay have a bigger impact on consumer surplus.

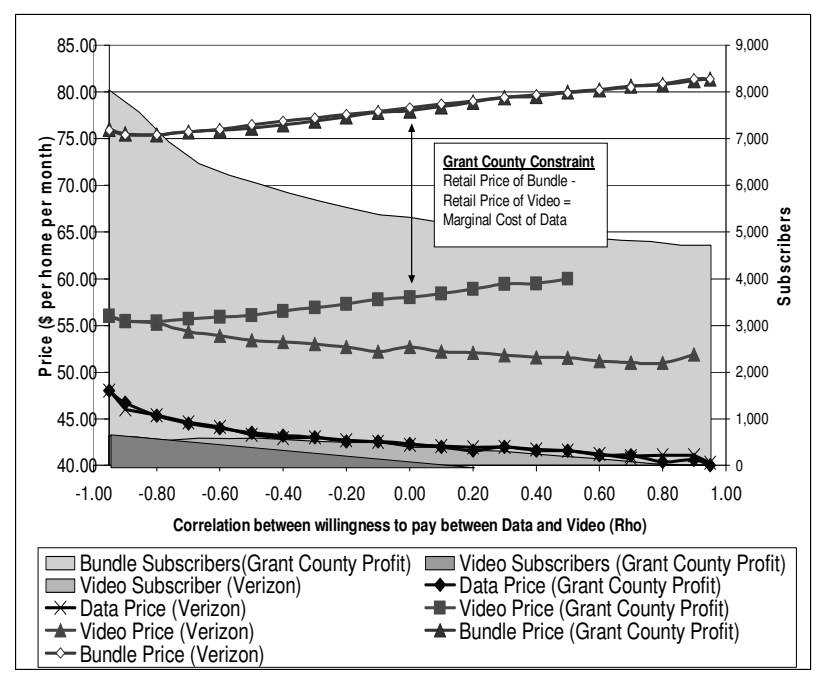

Figure 7. Comparison between 'Verizon' and 'Grant County Profit' (Prices and Number of Subscribers)

'Verizon' has marginally higher profit (about $\$ 0.10$ per home passed per month for $-0.75<\rho<0.75$ ) vis-à-vis 'Grant County Profit' due to its greater ability to price discriminate. For reasons already stated, for $\rho$ greater than 0.75 , the profits generated by 'Verizon' and 'Grant County Profit' are almost identical. For $\rho$ less than -0.75 , the similarity in profit outcomes is due to the similarity in the price of the video service for both industry structures ${ }^{5}$ (though the movement of the video price is in opposite directions - that is, 'Verizon's video price is decreasing and 'Grant County Profit's video price is increasing with increasing $\rho$ ). When $\rho$ is closer to -1 , both 'Verizon' and 'Grant County Profit' are able to serve a significantly higher number of subscribers resulting in higher profit and higher total welfare compared to the scenarios where $\rho$ is closer to +1 .

The marginal difference in profit between 'Grant County Profit' and 'Verizon' (of only around $\$ 0.10$ per subscriber per home passed) for the base case is a consequence of the cost structure of the industry. Recall that the marginal cost of supplying the bundle is less than the sum of the marginal costs of providing the individual services. This ensures that for a normal ${ }^{6}$ distribution of willingness to pay, most of the

\footnotetext{
${ }^{5}$ The value of $\rho(=-0.75)$ at which the video prices of both industry structures is equal appears to be influenced by our choice of model parameters. For $\rho>$ 0.75 the 'Verizon' video price is decreasing while the 'Grant County Profit' video price is increasing.

${ }^{6}$ We have also investigated uncorrelated uniform and exponential distributions of willingness to pay with little change in our conclusions.
}

extractable economic surplus ${ }^{7}$ lies in the bundle vis-à-vis the individual services. Since the wholesale retail split interferes with the ability of the wholesaler to extract the surplus associated with the video service (and not the bundle) the difference in profits is not very significant as the extractable economic surplus associated with the video service, especially for our choice of parameters, is modest.

Extending the above argument, the difference in profit between 'Verizon' and 'Grant County Profit' should increase with an increase in extractable economic surplus associated with the video service. The extractable surplus associated with the video service increases with (a) increase in mean willingness to pay for video service $\left(\mu_{2}\right)$, (b) decrease in mean willingness to pay for data service $\left(\mu_{1}\right)$, (c) decrease in the incremental cost of provisioning video service $\left(C_{2}\right)$ and $(\mathrm{d})$ increase in the incremental cost of provisioning data service $\left(C_{1}\right)$. Figure 8 shows how the difference in profit varies with different values for $\mu_{1}$ keeping the other model parameters fixed at $F=\$ 50,000$ per month, $C_{0}=\$ 8$ per home per month, $C_{1}=\$ 10$ per home per month, $C_{2}=\$ 30$ per home per month and $\mu_{2}=\$ 45$ per home per month. As expected the extractable surplus associated with the video service is the highest for the lowest value of $\mu_{1}(=\$ 15$ per home per month) and by the same token the difference between the profit of 'Verizon' and 'Grant County Profit' is maximum (about $\$ 0.30$ per home passed per month) for this value of $\mu_{1}$. For $\mu_{1}=\$ 25$ per home per month, the difference in profits turns out to be about $\$ 0.10$ per home passed per month, while the profits are almost identical for $\mu_{1}=\$ 35$ per home per month.

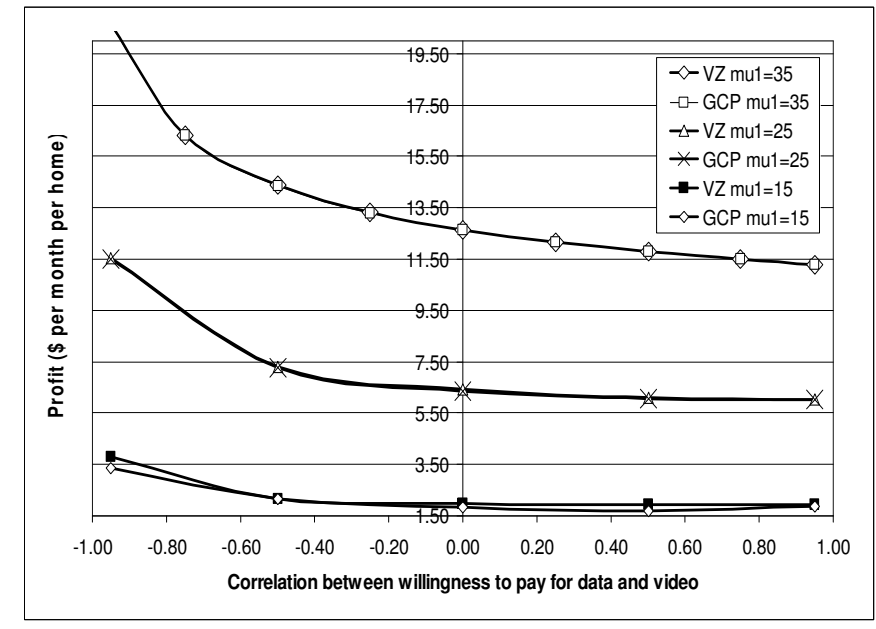

Figure 8. Comparing profits (per home passed) of 'Verizon' and 'Grant County Profit' for different values of $\mu_{1}$.

\footnotetext{
${ }^{7}$ By extractable surplus we refer to the area between the supply and the demand curves. Alternatively, this is also the maximum profit that can be extracted by a firm that has the ability to engage in first degree price discrimination. Notice that for the bundle, the mean willingness to pay is $\mu_{1}+$ $\mu_{2}$, but the cost of supplying the bundle is $C_{0}+C_{1}+C_{2}$, this ensures that majority of the extractable surplus resides in the bundle.
} 


\section{CONCLUSION, CAVEATS AND FUTURE WORK}

We conclude that for a bi-variate correlated normal distribution of willingness to pay for data and video services, the supply side economics of the FTTP industry (i.e., the marginal cost of provisioning the bundle is less than the sum of the marginal cost of provisioning the individual services) ensures that the bulk of the extractable economic surplus resides in the bundle and not in the individual services. Since the wholesale-retail split interferes with wholesaler's ability to extract economic surplus only from an individual service (video service) and not from the bundle, the implications (reduction in a wholesaler profits vis-à-vis profits of a vertically integrated entity) are modest, at best. Thus, we should expect that a wholesaler can readily realize sufficient revenue from wholesale services to pay for the network infrastructure. Contrary to the assertions of some current providers, our simplified model shows that it is not necessary to vertically integrate and exclude service level competitors in order to generate sufficient revenue to cover an investment in FTTP infrastructure.

The retail market (in the wholesale-retail split industry structures) is assumed to be perfectly contestable and competitive. However, we know that there are entry barriers, especially for the video business where a retailer needs to construct a video head end and get access to content. This will most likely result in oligopolistic competition (with the extreme case of a retail monopoly) that could cause (some degree of) double marginalization, thereby reducing welfare relative to a perfectly competitive retail market. Finally, we assume the incremental costs, $C_{1}$ and $C_{2}$, are the same in both vertically integrated and competitive retail cases. Also, we assume layer 2 costs, $C_{0}$, are the same whether supplied competitively (in the case of 'Stockholm') or by the wholesaler. Competition should drive down all these costs relative to the vertically integrated case.

.If we relax our assumption that the wholesaler sells only symmetric capacity, it is easy to see the wholesaler can indeed price discriminate to the same extent as a vertically integrated entity by selling a video capability that has high downstream bandwidth (say $4 \mathrm{Mbps}$ ) but very low upstream bandwidth (say a few Kbps that would be insufficient to support broadband data and severely compromise the broadband data experience). In general, there may be other technical choices that a wholesaler can make that would increase its ability to price discriminate, such as pricing separately for multicast capability used for video service.

The next logical step in this research is to come closer to the 'real world' by relaxing the assumption that every home takes voice and consider a 3-service model. Vertically integrated entities "Verizon" and "Bristol" can now sell seven 8 possible product bundles - (i) voice, (ii) video, (iii) data, (iv) voice and video, (v) voice and data, (vi) video and data, and

\footnotetext{
${ }^{8}$ In a world where a broadband data connection allows a home to purchase VoIP services from firms like Vonage, a vertically integrated entity like "Verizon" can sell only five product bundles (i) data (or data-voice bundle) service (ii) video service (iii) voice service (iv) video-voice bundle service and (v) data-video-voice bundle (or data-video bundle) service
}

(vii) voice, video and data. However, due to service arbitrage ${ }^{9}$, lit wholesalers - "Grant-County-Profit" and "Grant-CountyWelfare" can sell only four bundles; namely, (i) voice capability, (ii) video capability, (iii) voice- data bundle capability and (iv) voice-video-data bundle capability. From the intuition that we developed from our 2-service model, the supply side economics will most likely ensure that a large fraction of the economic surplus resides in the triple play bundle and one would expect our thesis to hold. Nevertheless, it will be interesting to see what the implications of the wholesale retail split are in the 3-service case, especially when one can expect the video-voice bundle to also have a reasonable amount of economic surplus that a wholesaler may find difficult to extract.

Finally, as we examine the implications of how the wholesale retail split imposes pricing inflexibility on the wholesaler (by interfering with its ability to price discriminate), we must recognize that the network owner may not be a monopolist in many markets. The final step in this research should seek to understand the implications of a wholesale retail split in the presence of inter modal competition with an incumbent (say, in the form of a Cable MSO), where the FTTP network owner will have lower pricing flexibility to start with.

\section{REFERENCES}

[1] A. Banerjee and M. Sirbu, "Towards Technologically and Competitively Neutral Fiber to the Premise (FTTP) Infrastructure", Broadband Services: Business Models and Technologies for Community Networks, I. Chlamtac, A. Gumaste \& S. Czabo (eds)., John Wiley 2005.

[2] H. Varian, "Price Discrimination", Handbook of Industrial Organization, Volume I, Elsevier Science Publisher, 1989

[3] W. J. Adams and J. L. Yellen, "Commodity Bundling and the Burden of Monopoly", The Quarterly Journal of Economics, Volume 90, No. 3, 1976

[4] R. Schmalensee, "Pricing of Product Bundles", Journal of Business, Volume 57, Number 1, 1984.

[5] J. Xie and M. Sirbu, "Price Competition and Compatibility in Presence of Positive Demand Externalities", Management Science, Volume 41, Number 5, May 1995.

[6] Bivariate Normal Distribution, available at http://www.math.wolfram.com

[7] Frigo, N.J., Iannone, P.P., and Reichmann, K.C., "A View of Fiber to the Home Economics," IEEE Comm Mag, 42,8, Aug 2004, pp. S16-S23.

[8] Abanesius, C., "Bristling over Muni Broadband in a Town Divided," National Journal's Insider Update, http://www.njtelecomupdate.com/lenya/telco/live/tbKXJR1123878279840.html Visited Sept 3, 2005

\footnotetext{
${ }^{9}$ As an example of service arbitrage, the wholesaler cannot sell 'voice and video capability' because the bandwidth associated with this offering permits the retailer to use it to sell a voice, video and data triple play bundle to the subscriber.
} 